\title{
Antibiotics resistance of extended spectrum beta lactamases uropathogenic Escherichia coli in Peshawar-Pakistan
}

\author{
Anees Muhammad ${ }^{1}$, Nasir Ali ${ }^{1}$, Shaukat $\mathrm{Ali}^{2}$, Syed Luqman Shuaib ${ }^{3}$, \\ Irfan Qadir Afridi ${ }^{4}$, Waqar Saeed ${ }^{2}$ and Muhammad Jaseem Khan ${ }^{5^{*}}$ \\ 1. Department of Medical Laboratory Technology, College of Medical Technology, Medical Teaching Institution, \\ Bacha Khan Medical College, Mardan-Pakistan \\ 2. Department of MLT, The University of Haripur, Haripur-Pakistan \\ 3. Pathology (Microbiology), Khyber Medical College/ Khyber Teaching Hospital, Peshawar-Pakistan \\ 4. Khyber Teaching Hospital, Medical Teaching Institution, Peshawar-Pakistan \\ 5. Department of MLT, Institute of Paramedical Sciences, Khyber Medical University, Peshawar-Pakistan \\ *Corresponding author's email: jaseem.leo@gmail.com \\ Citation \\ Anees Muhammad, Nasir Ali, Shaukat Ali, Syed Luqman Shuaib, Irfan Qadir Afridi, Waqar Saeed and Muhammad \\ Jaseem Khan. Antibiotics resistance of extended spectrum beta lactamases uropathogenic Escherichia coli in \\ Peshawar-Pakistan. Pure and Applied Biology. Vol. 9, Issue 3, pp1840-1848. \\ http://dx.doi.org/10.19045/bspab.2020.90196
}

Received: 06/02/2020 Revised: 24/04/2020 Accepted: 07/05/2020

Online First: 13/05/2020

\section{Abstract}

Urinary tract infection (UTI) is predominantly caused by Escherichia coli (E. coli). E. coli is the major extended-spectrum beta-lactamases (ESBL) producing uropathogenic bacteria, leading to serious complications and limiting therapeutic management. This study was undertaken to measure the proportion of ESBL producing and antibiotic susceptibility pattern of uropathogenic E. coli in Peshawar. During the study duration, a total of 214 isolates of uropathogenic E. coli with urine samples were collected from patients. The urine samples were inoculated on the recommended media for the growth of bacteria. The bacteria growth was identified based on clinical laboratory standard institute (CLSI) protocol. The antibiotic susceptibility testing (AST) was carried out for E. coli using various discs of antibiotics. The ESBL producing E. coli were identified by double discs diffusion method. Among the studied isolates, $46.3 \%$ were collected from male patients. The highest percentage of patients was identified in the age group of 21-30 years. The female patients were observed more resistant to antibiotics. Similarly, ESBL producing isolates were more resistant than non-ESBL producing isolates. The $E$. coli isolates were more resistant to few antibiotics including nalidixic acid, cefuroxime sodium, cephradine, and ofloxacin while least to imipenem, fosfomycin, and meropenem. Conclusively, MDR and ESBLs producing E. coli isolates were observed more challenging in the studied region. Therefore, the early identification of ESBL producing and MDR E. coli isolates may be helpful to prevent further resistance in patients and will be helpful for better management of UTIs. Keywords: Antibiotics; E. coli; ESBL; Multi-drug resistant; Peshawar

Introduction

Urinary tract infection (UTI) is the most

common nosocomial and community

bacterial infection and significantly responsible for health care cost and morbidity rate $[1,2]$. The un-diagnosed UTIs leads to damage renal structure and function (kidney failure) [3]. The UTIs are responsible for 
almost $35 \%$ of nosocomial infections $[4,5]$. UTIs are the prevalent infectious diseases affecting about 0.15 billion individuals worldwide each year. Affecting all age groups of both sexes unanimously but the proportion of females is more as compared to males [6, 7]. Among 100 healthy females, every 5th female is suffering from uncomplicated UTIs cases annually while about twenty percent of cases occurring in males [7].

The gram-negative bacteria are the most common etiological agent of UTIs [8] especially the Escherichia coli (E. coli) is identified as the predominant causative bacterial isolates responsible for more than $80 \%$ of uncomplicated UTIs cases [9]. Other gram-negative isolates like Klebsiella species (spp.), Pseudomonas spp., Proteus spp. and Enterobacter spp. and gram-positive isolates including

Staphylococcus

spp.,

Streptococcus spp. and Enterococcus spp. account from $5-15 \%$ of UTIs cases $[10,11]$.

The UTIs treatment is a challenging task due to antibiotics resistance but frequently treated with broad-spectrum fluoroquinolones (ciprofloxacin), cephalosporins (ceftazidime, cefepime) and aminoglycosides (gentamicin, amikacin). The fluoroquinolones are inhibitors of DNA topoisomerase and gyrase, cephalosporins are antibiotics to inhibits cell wall and aminoglycosides can inhibit the protein synthesis of bacteria [12]. Among these antibiotics, beta $(\beta)$ lactam antibiotics are frequently used as a therapeutic agent for UTIs particularly related to gram-negative bacterial infections [13].

The antibiotics resistance increased all over the world because of the production of extended beta spectrum lactamases (ESBL) producing by bacteria due to the frequent use of beta-lactam antibiotics in the last two decades [14-16]. The ESBL producing bacterial isolates are associated with greater morbidity and fatality than non-ESBL producer strain of E. coli [17]. The E. coli producing ESBL is possibly one of the important mechanisms of resistance to cephalosporins and penicillins which may be chromosomally mediated or plasmidmediated $\beta$-lactamases $[18,19]$. The treatment of $E$. coli with beta-lactam antibiotics in the presence of ESBL production is useless [20]. Moreover, the ESBL producing E. coli strains are usually related to multi-drug resistance (MDR). The coexistence of MDR and ESBL production worsens the condition and complicates the treatment particularly in developing countries [16].

The ESBL producing E. coli strains have been significantly determined from various regions of the world. Inter-regional antibiotics susceptibility variation is high in ESBL producing E. coli. It is important to known and understands the common ESBL producing bacteria such as E. coli for necessary management. Therefore, the current study was undertaken to find the percentage of uropathogenic E. coli producing ESBL and its antibiotic susceptibility profile in Peshawar, Pakistan.

\section{Materials and methods}

\section{Study design, duration and study setting}

This cross-sectional descriptive study was performed in the microbiology department of a well-reputed private laboratory (Pak Medical Center and Laboratory) in Peshawar from January to June 2019.

\section{Study population \\ Inclusion criteria}

The patients visited clinics were included in the study population. The patients suspected UTIs with symptoms such as an intense urge to urination; fever/chills, abdominal pain (back or lower), and burning micturition were included.

\section{Exclusion criteria}

TheUTI patients received antibiotic history in the last one week, have urinary catheterization, and samples without labeling or held for more than 2 hours without any 
preservative or at temperature $0-8^{\circ} \mathrm{C}$ were excluded from the study. The isolates were non-repetitive (single sample per patient).

\section{Ethical consideration}

The permission of the study was obtained from authorized committee members of the Pak Medical Center and Laboratory.

\section{Sample collection and processing}

The midstream fresh urine samples $(5-10 \mathrm{ml})$ were collected in screw-capped, sterile container aseptically and were further processed for culture and sensitivity within two hours of collection. The urine samples were streaked on cysteine lactose electrolyte deficient agar (CLED), MacConkey agar (MA) and Blood agar (BA) (Oxide, Ltd England) plates according to the clinical laboratory standard institute (CLSI) guidelines [21]. The culture Petriplates were aerobically incubated at $37^{\circ} \mathrm{C}$ for overnight. The culture plates were checked periodically for bacterial growth after 18-24 hours. The gram-negative bacteria were identified by the morphological appearance of colonies, characteristics of gram staining, and biochemical tests (lactose fermentation, triple sugar iron, indole reaction, and citrate utilization) as per CLSI protocol [21]. The $E$. coli growth with more than 10 colonyforming units per milliliter (ml) was considered clinically significant [22].

\section{Antibiotics susceptibility testing}

The antibiotic susceptibility testing for recovered $E$. coli isolates was performed on Mueller Hilton Agar (MHA) by Kirby Bauer disk diffusion method. A total of 18 commercially available antibiotic discs (Oxide, Ltd England) were used with their appropriate concentrations: amikacin (30ug), amoxicillin (10ug), augmentin: amoxicillinclavulanic acid $(30 \mu \mathrm{g})$, azithromycin $(15 \mu \mathrm{g})$,

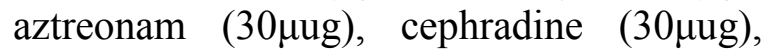
cefotaxime $(30 \mu \mathrm{g})$, cefoxitin $(30 \mu \mathrm{g})$, ceftazidime $(30 \mu \mathrm{g})$, ceftriaxone $(30 \mu \mathrm{g})$, cefuroxime $(30 \mu \mathrm{g})$, ciprofloxacin $(5 \mu \mathrm{g})$, fosfomycin $(50 \mu \mathrm{g})$, gentamicin $(10 \mu \mathrm{g})$, imipenem $(10 \mu \mathrm{g})$, meropenem $(10 \mu \mathrm{g})$, nalidixic acid $(30 \mu \mathrm{g})$, nitrofurantoin $(30 \mu \mathrm{g})$, norfloxacin $(10 \mu \mathrm{g})$, ofloxacin $(5 \mu \mathrm{g})$, piperacillin-tazobactam $\quad(40 \mu \mathrm{g}) \quad$ and cotrimoxazole: trimethoprimsulphamethoxazole $(25 \mu \mathrm{g})$. The zone of inhibition around antibiotics discs formed by E. coli was measured in millimeter ( $\mathrm{mm})$ and interpreted according to the CLSI standards. E. coli strain American Type Culture Collection (ATCC) 25922 was used as a reference control strain $[21,22]$.

\section{ESBL identification}

All the recovered $E$. coli strains were tested for phenotypic detection of ESBL production. The ESBL producing E. coli isolates were initially identified by a zone of inhibition of cephalosporins (cefotaxime, cefoxitin, ceftazidime, and ceftriaxone) which was the further process for confirmation and were verified as ESBL producer by double disc diffusion method (cephalosporins and monobactam). The clear zone of inhibition ( $\geq 5 \mathrm{~mm}$ ) of cephalosporins extended towards the aztreonamwas considered as ESBL producing E. coli. The E. coli strain was considered as multi-drug resistant if it was resistant to three or more antibiotics of various groups/classes of antibiotics [21, 22].

\section{Data analysis}

The data were analyzed by statistical package for social sciences (SPSS version 21). The frequency and percentages were calculated for all categorical variables such as gender, microorganism, and ESBL number. The mean and standard deviation was computed for the age of patients.

\section{Results and discussion}

During the study period, a total of 214 confirmed $E$. coli isolates were collected from urine samples with UTIs patients. Out of total (214), males were $46.3 \%(n=99)$ and females patients were $53.7 \%(\mathrm{n}=115)$. The prevalence of females $(53.7 \%)$ was more than male $(46.3 \%)$ in present findings. This 
predominance in female to male is concordance the study of Polse et al. from Iraq [23]. The study conducted by Jamil et al., from Swabi, Pakistan revealed $66.3 \%$ prevalence in females [24]. Our finding also supports other previously conducted studies with the same proportion of female to male urinary tract infections cases caused by $E$. coli $[14,15]$. The high number of female patients could be due to anatomic posture of females including urethral opening, the proximity of the urethra to anus, short urethra, and during pregnancy physiologically leads to urinary tract infections [16].

The mean age of male patients was $45.1 \pm 19.4$ years in which majority male patients were noted in 31-40 years of age group (19.2\%, $\mathrm{n}=19)$, followed by $21-30$ years $(18.2 \%$, $\mathrm{n}=18)$ and $51-60$ years $(17.2 \%, \mathrm{n}=17)$. On the other hand, frequent cases of females were observed in the age group of 21-30 years $(24.3 \%, \mathrm{n}=28)$, followed by $51-60$ years and $61-70$ years $(16.5 \%, \mathrm{n}=19$ each).The highest number of urinary tract infections cases found in the age group of 21-30 years with $21.5 \%(n=46)$ (Table 1). It is observed in our study that UTIs caused by E. coli were more frequently occur in 11-50 years of age with the highest number of cases identify in the age group of 21-30 years (21.5\%). Our findings in line with the results of different researchers which reveals a high prevalence of UTI caused by E. coli at the age of 21-30 years $[12,17]$. The frequent cases at a young age maybe because of sexual activity and using certain kinds of contraceptives can raise the risk at the age of 21-30 years [18]. The present study shows that $88.3 \%(n=189)$ strains were resistant to three or more classes of antibiotics and are known as the multidrug resistant in which highest proportion of patients were occurred in the age group of 2130 years $(22.2 \%, \mathrm{n}=42)$, followed by $51-60$ years $(16.9 \%, \mathrm{n}=32)$ and $31-40$ years $(15.3 \%$, $\mathrm{n}=29$ ) (Table 1). The highest number of
MDR found in ESBL producing E. coli infected patients with $90.1 \% \quad(\mathrm{n}=145)$ as compared to non-ESBLs $(83.0 \%, \quad(n=44)$ (Table 2). Furthermore, a large proportion of MDR isolates were observed in males $(89.9 \%, \mathrm{n}=89)$ than female patients $(87 \%$, $\mathrm{n}=100$ ) (Table 3). Almost similar MDR prevalence $(81.9 \%)$ was reported by Shakhatreh et. al from Jordan [25]. A previous study reports showed $82 \%, 65.5 \%$ and $65 \%$ MDR isolates of uropathogenic $E$. coli $[14,20,23]$. Additionally, the current study shows that $76.7 \%$ of MDR isolates identified in ESBL producing E. coli. Ullah et al., reported $83 \%$ MDR isolates in ESBL producing E. coli [26]. Moreover, this study indicated that drug resistance was high among ESBL producing $E$. coli than the nonESBL producer. The production of betalactamases enzymes may be a plasmid or chromosomal origin. Plasmid origin is occurred by transferring genetic materials from one to other organisms which leads to antibiotics resistance [25]. Therefore, the MDR isolates are probable to be common in E. coli producing ESBL isolates.

The ESBLs phenotype was detected in $75.2 \%$ $(n=161) E$. coli isolates in which highest number of isolates observed in age group of 21-30 years $(22.3 \%, \mathrm{n}=36)$, followed by 61 70 years $(19.8 \%, \mathrm{n}=32)$ and $51-60$ years $(13.6 \%, \mathrm{n}=22)$ (Table 1). The percentage of ESBLs producing $E$. coli was found higher in males $(76.8 \%, \mathrm{n}=76)$ as compared to females (73.9\%, $\mathrm{n}=85$ ) (Table 3). The percentage of E. coli producing ESBL was $75.2 \%$ which is similar to the study conducted at by Ahn et al., and Al-Mously, et al. which is $71.7 \%$ and $75.1 \%$ respectively $[27,28]$ while high with the study conducted at Jordan by Shakhatreh et. al $(49.9 \%)$ [25]. In contrast to the report of Jamil et al., where ESBLs producing E. coli proportion is $33.3 \%$ [24]. Another study reported $64.9 \%$ ESBL producing E. coli isolates which nearly similar to our study [23]. Ahmed et al., from Pakistan reported 
$53.4 \%$ ESBL producing isolates of E. coli which is lower than the present study [29]. Additionally, multi-drug resistant and ESBL producing $E$. coli cases was high in male
$(\mathrm{MDR}=46.3 \%$ \& $\mathrm{ESBL}=47.2 \%)$ as compared to female patients (MDR $=53.7 \% \quad \&$ $\mathrm{ESBL}=52.8 \%$ ) that agreed with previous findings [27].

Table 1. According to the age and gender of patients, MDR, and ESBL producing isolates of E.coli are distributed

\begin{tabular}{|c|c|c|c|c|c|}
\hline Age (Years) & Male \% (n) & Female \% (n) & Total \% (n) & MDR \% (n) & ESBL \% (n) \\
\hline $0-10$ & $01.0(01)$ & $0.90(01)$ & $0.90(02)$ & $1.10(02)$ & $1.10(02)$ \\
\hline $11-20$ & $09.1(09)$ & $11.4(13)$ & $10.3(22)$ & $10.6(20)$ & $10.5(17)$ \\
\hline $21-30$ & $18.2(18)$ & $24.3(28)$ & $21.5(46)$ & $22.2(42)$ & $22.3(36)$ \\
\hline $31-40$ & $19.2(19)$ & $10.4(12)$ & $14.5(31)$ & $15.3(29)$ & $13.0(21)$ \\
\hline $41-50$ & $12.1(12)$ & $13.0(15)$ & $12.6(27)$ & $13.8(26)$ & $11.7(19)$ \\
\hline $51-60$ & $17.2(17)$ & $16.5(19)$ & $16.8(36)$ & $12.2(23)$ & $13.6(22)$ \\
\hline $61-70$ & $15.1(15)$ & $16.5(19)$ & $15.9(34)$ & $16.9(32)$ & $19.8(32)$ \\
\hline$>70$ & $08.1(08)$ & $7.0(08)$ & $07.5(16)$ & $7.90(15)$ & $08.0(13)$ \\
\hline Total & $46.3(99)$ & $53.7(115)$ & $100(214)$ & $88.3(189)$ & $75.2(161)$ \\
\hline
\end{tabular}

The E. coli showed higher resistance to nalidixic acid (93.9\%), followed by cefuroxime sodium $(93.5 \%)$, cephradine $(90.2 \%)$ and ofloxacin $(90.2 \%)$ while least resistance was found against to imipenem (25.7\%), fosfomycin (36.0\%) and meropenem (38.8\%). However, antibiotics susceptibility analysis illustrates that all ESBL producing E. coli strains have a higher resistance to almost all tested antibiotics except cephradine and gentamicin as compared to non-ESBL strains (Table 2).

Table 2. Comparison of antibiotic resistance $E$. coli isolates between ESBL and Non-ESBL

\begin{tabular}{|c|c|c|c|}
\hline Antibiotics & ESBL \% $(\mathbf{n = 1 6 1})$ & Non-ESBL \%(n)53 & Total Resistance \%(n)214 \\
\hline MDR \% (n) & $76.7(145)$ & $23.3(44)$ & $88.3(189)$ \\
\hline Amikacin & $60.9(98)$ & $47.2(25)$ & $53.7(115)$ \\
\hline Augmentin & $87.6(141)$ & $77.4(41)$ & $83.6(179)$ \\
\hline Aztreonam & $100(161)$ & $60.4(32)$ & $79.9(171)$ \\
\hline Ceftazidime & $93.2(150)$ & $67.9(36)$ & $84.6(181)$ \\
\hline Cefotaxime & $93.8(151)$ & $75.5(40)$ & $86.4(185)$ \\
\hline Ceftriaxone & $94.4(152)$ & $75.5(40)$ & $88.3(189)$ \\
\hline Cefuroxine sodium & $96.3(155)$ & $88.7(47)$ & $93.5(200)$ \\
\hline Cephradine & $93.2(150)$ & $94.3(50)$ & $90.2(193)$ \\
\hline Ciprofloxacin & $91.9(148)$ & $83.0(44)$ & $84.6(181)$ \\
\hline Fosfomycin & $32.9(53)$ & $26.4(14)$ & $36.0(77)$ \\
\hline Gentamicin & $68.9(111)$ & $71.7(38)$ & $68.7(147)$ \\
\hline Imipenam & $31.7(51)$ & $18.9(10)$ & $25.7(55)$ \\
\hline Meropenam & $42.2(68)$ & $24.5(13)$ & $38.8(83)$ \\
\hline Nalidix acid & $96.9(156)$ & $88.7(47)$ & $93.9(201)$ \\
\hline Nitrofurantoin & $69.6(112)$ & $60.4(32)$ & $70.6(151)$ \\
\hline Ofloxacin & $93.8(151)$ & $83.0(44)$ & $90.2(193)$ \\
\hline Piperacillin/tazobactam & $64.6(104)$ & $41.5(22)$ & $59.3(127)$ \\
\hline Sulbactum/Cefoperazone & $63.4(102)$ & $45.3(24)$ & $58.4(125)$ \\
\hline
\end{tabular}


It was also observed that approximately all antibiotics besides few (ceftriaxone, gentamicin, nalidixic acid, and nitrofurantoin) have a better response to uropathogenic E. coli in males as compared to female. The carbapenem and fosfomycin were most effective against uropathogenic $E$. coli while the least effective was nalidixic acid, cefuroxime sodium, cephradine, and ofloxacin. Our finding was consistent with the previous studies that reported high resistance to nalidixic acid and cefuroxime $[14,19]$. Sharma et al., from India mentioned that uropathogenic E. coli showed higher sensitivity to imipenem that is consistent with the finding of this study [28]. In a recent study, researchers revealed similar imipenem sensitivity as the current study $[13,17,23]$.
Furthermore, this study showed that almost all tested antibiotics were less resistant against non-ESBLs than ESBLs producing $E$. coli except cephradine and gentamicin. A similar finding was reported by the previous report that is agreed with the present study [17].

In this study, the male showed higher resistance than female to various antibiotics including amikacin, augmentin, aztreonam, ceftazidime, cefotaxime, cefuroxime sodium, cephradine, ciprofloxacin, fosfomycin, imipenem, meropenem, ofloxacin, piperacillin/ tazobactam, and sulbactam/ cefoperazone whereas few antibiotics (ceftriaxone, gentamicin, nalidixic acid, and nitrofurantoin) showed higher resistance in female as compared to male (Table 3 ).

Table 3. Comparison of antibiotic resistance between male and female infected patients

\begin{tabular}{|c|c|c|}
\hline Antibiotics & Male\% (n) 99 & Female \% (n) 115 \\
\hline MDR & $46.3(89)$ & $53.7(100)$ \\
\hline ESBL & $47.2(76)$ & $52.8(85)$ \\
\hline Amikacin & $59.6(59)$ & $55.6(64)$ \\
\hline Augmentin & $85.9(85)$ & $84.3(97)$ \\
\hline Aztreonam & $91.9(91)$ & $87.8(101)$ \\
\hline Ceftazidime & $87.9(87)$ & $86.1(99)$ \\
\hline Cefotaxime & $92.9(92)$ & $86.1(99)$ \\
\hline Ceftriaxone & $88.9(88)$ & $90.4(104)$ \\
\hline Cefuroxime sodium & $99.0(98)$ & $92.2(106)$ \\
\hline Cephradine & $96.0(95)$ & $91.3(105)$ \\
\hline Ciprofloxacin & $90.9(90)$ & $87.8(101)$ \\
\hline Fosfomycin & $32.3(32)$ & $30.4(35)$ \\
\hline Gentamicin & $59.6(59)$ & $78.3(90)$ \\
\hline Imipenem & $36.4(36)$ & $19.1(25)$ \\
\hline Meropenem & $43.4(43)$ & $33.0(38)$ \\
\hline Nalidixic acid & $92.9(92)$ & $95.7(110)$ \\
\hline Nitrofurantoin & $65.7(65)$ & $77.4(89)$ \\
\hline Ofloxacin & $96.0(95)$ & $87.0(100)$ \\
\hline Piperacillin/tazobactam & $61.6(61)$ & $56.5(65)$ \\
\hline Sulbactum/Cefoperazone & $94.9(94)$ & $53.9(62)$ \\
\hline
\end{tabular}

This study has some limitations; the study was limited to a single center with a small sample size and also could not confirm the ESBLs producing E. coli at a molecular level. This study does not reveal the picture of the whole country; hence longitudinal and prospective study is necessary to a covere wide range of population to get a geographical, ethnic, and seasonal variation of $E$. coli and their susceptibility pattern of 
antibiotics. Although, there were few limitations we believe that this study provides better information for selecting an empirical therapy and will help clinicians in the future to control the continuous resistance of antibiotics.

The higher number of MDR and ESBL producing isolates in our regions is a great public problem. This indicates that continuous monitoring, infection control measurement, and proper administration of antibiotics are required to reduce the frequency of ESBL. Additionally, antibiotics should be selected for the treatment of urinary tract infections based on culture and sensitivity reports of urine.

\section{Conclusion}

Our study highlights the frequency of ESBL producing $E$. coli along with MDR isolates and their antibiotics susceptibility pattern in our regions. The higher percentages of MDR were observed in male and ESBL producing E. coli isolates. These findings suggest further molecular studies regarding the MDR and ESBL strains of E. coli. Moreover, it is strongly recommended that appropriate antibiotics therapy and periodical surveillance can play a better role in the control of the antibiotics resistance strains along with ESBL producers. Furthermore, novel drugs and their selection against particular isolates are need of today.

\section{Authors' contributions}

Conceived and designed the experiments: A Muhammad, S Ali \& MJ Khan, Performed the experiments: A Muhammad, S Ali, SL Shuaib, IQ Afridi \& W Saeed, Analyzed the data: A Muhammad, N Ali \& W Saeed, Contributed materials/ analysis/ tools: N Ali, W Saeed \& MJ Khan, Wrote the paper: A Muhammad, N Ali, S Ali \& SL Shuaib Critical Review: N Ali, SL Shuaib, IQ Afridi and MJ Khan

\section{Acknowledgment}

We would like to thank Mr. Khalid Khan, Microbiologist, and Incharge of Pak Medical
Laboratory for his support, encouragement, cooperation and permission given for data collection.

\section{References}

1. Sharifi Y, Hasani A, Ghotaslou R, Naghili B, Aghazadeh M, Milani M \& Bazmany A (2013). Virulence and antimicrobial resistance in enterococci isolated from urinary tract infections. Adv Pharm Bull 3(1): 197201.

2. Gupta K, Hooton TM \& Stamm WE (2001). Increasing antimicrobial resistance and the management of uncomplicated community-acquired urinary tract infections. Ann Intern Med 135(1): 41-50.

3. Murray PR, Baron EJ, Jorgense JH, Pfaller MA \& Yolken RH (2003). Manual of Clinical Microbiology. American Society for Microbiology. 8th Ed. Washington DC. Press, 2003. 2322 pp.

4. Kariuki S, Revathi G, Corkill J, Kiiru J, Mwituria J, Mirza N \& Hart CA (2007). Escherichia coli from communityacquired urinary tract infections resistant to fluoroquinolones and extendedspectrum beta-lactams. The $J$ Infect Dev 1(03): 257-262.

5. Taneja N, Chatterjee SS, Singh M, Singh S \& Sharma M (2010). Pediatric urinary tract infections in a tertiary care center from north India. Indian $J$ Med Res 131(1): 101-106.

6. Stamm WE \& Norrby SR (2001). Urinary tract infections: disease panorama and challenges. $J$ Infect Dis 183(1): 1-4.

7. Sood S \& Gupta R (2012). Antibiotic resistance pattern of communityacquired uropathogens at a tertiary care hospital in Jaipur, Rajasthan. Indian $J$ Community Med 37(1): 39-44.

8. Weinstein RA, Gaynes R, Edwards JR \& National Nosocomial Infections 
Surveillance System. (2005). Overview of nosocomial infections caused by gram-negative bacilli. Clin Infect Dis 41(6): 848-854.

9. Piatti G, Mannini A, Balistreri M \& Schito AM (2008). Virulence factors in urinary Escherichia coli strains: phylogenetic background and quinolone and fluoroquinolone resistance. J Clin Microbiol 46(2): 480-487.

10. Ilyas M, Ahmad S, Khurram M, Mazhar K \& Sajid A (2014). Susceptibility pattern of extended spectrum-lactamases positive Escherichia coli isolated from a tertiary care hospital of Peshawar, Pakistan. World Appl Sci J 30(3): 253257.

11. Akram M, Shahid M \& Khan AU (2007). Etiology and antibiotic resistance patterns of community-acquired urinary tract infections in JNMC Hospital Aligarh, India. Ann Clin Microbiol Antimicrob 6(1): 1-7.

12. Trevor AJ, Katzung BG, Masters SB \& Kruidering HM (2010). Pharmacology examination \& board review. 11th Ed. New York: McGraw-Hill Medical.

13. Raei F, Eftekhar F \& Feizabadi MM (2014). Prevalence of quinolone resistance among extended-spectrum $\beta$ lactamase producing uropathogenic Klebsiella pneumoniae. Jundishapur $J$ Microbiol 7(6): 1-5.

14. Osthoff M, McGuinness SL, Wagen AZ $\&$ Eisen DP (2015). Urinary tract infections due to extended-spectrum beta-lactamase-producing Gramnegative bacteria: identification of risk factors and outcome predictors in an Australian tertiary referral hospital. Int $J$ of Infec Dis 34: 79-83.

15. Cantas L, Suer K, Guler E \& Imir T (2016). High emergence of ESBLproducing E. coli cystitis: time to get smarter in Cyprus. Front Microbiol 6: 17.
16. Kim MH, Lee HJ, Park KS \& Suh JT (2010). Molecular characteristics of extended-spectrum $\beta$-lactamases in Escherichia coli and Klebsiella pneumoniae and the prevalence of qnr in extended-spectrum $\beta$-lactamase isolates in a tertiary care hospital in Korea. Yonsei Med J 51(5): 768-774.

17. Kim YK, Pai H, Lee HJ, Park SE, Choi EH, KimJ \& Kim EC (2002). Bloodstream infections by extendedspectrum $\quad \beta$-lactamase-producing Escherichia coli and Klebsiella pneumoniae in children: epidemiology and clinical outcome. Antimicrob Agents Chemother 46(5): 1481-1491.

18. Chaudhary U \& Aggarwal R (2004). Extended spectrum-lactamases (ESBL)An emerging threat to clinical therapeutics. Indian $J \quad$ Med Microbiol 22(2): 75-80.

19. Bradford PA (2001). Extended-spectrum $\beta$-lactamases in the $21 \mathrm{st}$ century: characterization, epidemiology, and detection of this important resistance threat. Clin Microbiol Rev 14(4): 933951.

20. Hyle EP, Lipworth AD, Zaoutis TE, Nachamkin I, Fishman NO, Bilker WB \& Lautenbach E (2005). Risk factors for increasing multidrug resistance among extended-spectrum $\quad \beta$-lactamaseproducing Escherichia coli and Klebsiella species. Clin Infect Dis 40(9): 1317-1324.

21. Othman IEI (2016). Microbiological Assessment of Quality Control in Governmental Laboratories in Khartoum State. B.Sc. Thesis, Dept of Microbio., Sudan Univ. of Sci \& Tech., Sudan.

22. Clinical and Laboratory Standards Institute (2014). Performance Standards for Antimicrobial Susceptibility Testing; Twenty-Fourth Informational Supplement. 13(1): M100-S24. 
23. Polse R, Yousif S, \& Assafi M (2016). Prevalence and antimicrobial susceptibility patterns of uropathogenic E. coli among people in Zakho, Iraq. Int J Res Med Sci 4(4): 1219-1223.

24. Jamil J, Haroon M, Sultan A, Khan MA, Gul N \& Kalsoom (2018). Prevalence, antibiotic sensitivity, and phenotypic screening of ESBL/MBL producer $E$. coli strains isolated from urine; District Swabi, KP, Pakistan. J Pak Med Assoc 68(11): 1704-1707.

25. Shakhatreh MA, Swedan SF, Ma'en A \& Khabour OF (2019). Uropathogenic Escherichia coli (UPEC) in Jordan: Prevalence of urovirulence genes and antibiotic resistance. J King Saud Univ Sci 31(4): 648-52.

26. Ullah F, Malik S \& Ahmed J (2009). Antibiotic susceptibility pattern and ESBL prevalence in nosocomial Escherichia coli from urinary tract infections in Pakistan. Afr $J$ Biotechnol 8(16): 3921-3926.

27. Sharma N, Gupta A, Walia G \& Bakhshi $R$ (2016). Pattern of antimicrobial resistance of Escherichia coli isolates from urinary tract infection patients: a three year retrospective study. J Appl Pharm Sci 6(01): 062-065.

28. Ahn ST, Kim SW, Kim JW, Park HS, Moon DG \& Oh MM (2019). Does urinary tract infection caused by extended-spectrum $\quad \beta$-lactamaseproducing Escherichia coli show same antibiotic resistance when it recurs. $J$ Infect Chemother 25(7): 498-502.

29. Ahmed I, Sajed M, Sultan A, Murtaza I, Yousaf S, Maqsood B \& Anees M (2015). The erratic antibiotic susceptibility patterns of bacterial pathogens causing urinary tract infections. EXCLI J 14: 916-925. 